\title{
A CFD model of hemodynamics in hemodialysis vascular access
}

\author{
A. Ciandrini ${ }^{1}$, P. G. Walker ${ }^{2}$, M. K. Kolandavel ${ }^{2}$, C. A. Lodi ${ }^{3}$, \\ R. Galato ${ }^{4}$ \& S. Cavalcanti ${ }^{1}$ \\ ${ }^{I}$ Department of Electronics, Computer Science and Systems, \\ University of Bologna, Italy \\ ${ }^{2}$ School of Mechanical Engineering, University of Leeds, U.K. \\ ${ }^{3}$ R\&D Monitor Division, Gambro Dasco S.p.A., Medolla (MO), Italy \\ ${ }^{4}$ Nephrology Division, San Carlo Clinic, Paderno Dugnano (Mi), Italy
}

\begin{abstract}
Hemodialysis treatment requires a patient's blood circulation to be connected to artificial kidney extracorporeal (EC) circulation through a vascular access. In chronic patients, the vascular access is normally created by an artero-venous (AVF) fistula, where part of the peripheral arterial blood flows along a venous vessel. The connection is made by inserting two needles into the venous vessel. Blood is drawn to the EC along the arterial needle and is returned to the patient along the venous needle. In this study, we simulated the hemodynamics of vascular access with particular focus on the region downstream to the venous needle in order to analyze the influence of return flow $(\mathrm{Qb})$ on blood circulation. A three dimensional CFD model of vascular access circulation was developed and various blood flow conditions were simulated. Simulation results predicted a critical circulation downstream to the needle tip due to the confluence between the return flow and the access flow. A vortex recirculation was evident in this region. Vortex extension appeared only for $\mathrm{Qb}$ values higher than $40 \%$ of the access flow and was limited to a zone few centimetres from the needle tip in the downstream direction. CFD simulations allowed a detailed study of the complex hemodynamics in the vascular access region surrounding the inserted needle which would otherwise be difficult to measure experimentally.

Keywords: CFD model, hemodialysis, vascular access, vortex flow, shear stress.
\end{abstract}




\section{Introduction}

Vascular access is usually considered as one of the most critical aspects of the hemodialysis treatment. In fact, vascular access failure represents a major cause of morbidity and hospitalisation for dialysis patients with a high cost impact on the public health [1]. At present, vascular access for hemodialysis is a hot international research topic. Vascular access devices may often create dangerous conditions in patients through vascular tissue damage and the failure of vascular access $[2,3]$. Cannula design to suit the requirements of individual patients can prevent the failure of access and will be a significant technological advancement in hemodialysis. This is possible by predicting the complex haemodynamics of a typical vascular access for dialysis patients, namely the artero-venous fistula (AVF). As an alternative to the in vitro studies a recent vascular access study used computational fluid dynamics (CFD) to predict the local hemodynamics in the right atrium in the presence of a Niagara catheter [4]. CFD is particularly useful because it allows a detailed study of the complex haemodynamics in different geometric configurations with different flow conditions. If properly used, it can provide a faithful prediction of in vivo blood flow situation. Hence, the aim of this study is to quantify the hemodynamics of a typical vascular access for dialysis patients, namely the arterious-venous fistula (AVF) by using CFD. In particular, a CFD model of the AVF near the point of re-infusion was developed.

\section{Materials and methods}

\subsection{Geometric model and materials}

A schematic geometric model used to simulate the blood re-infusion in an AVF is represented in Fig. 1.

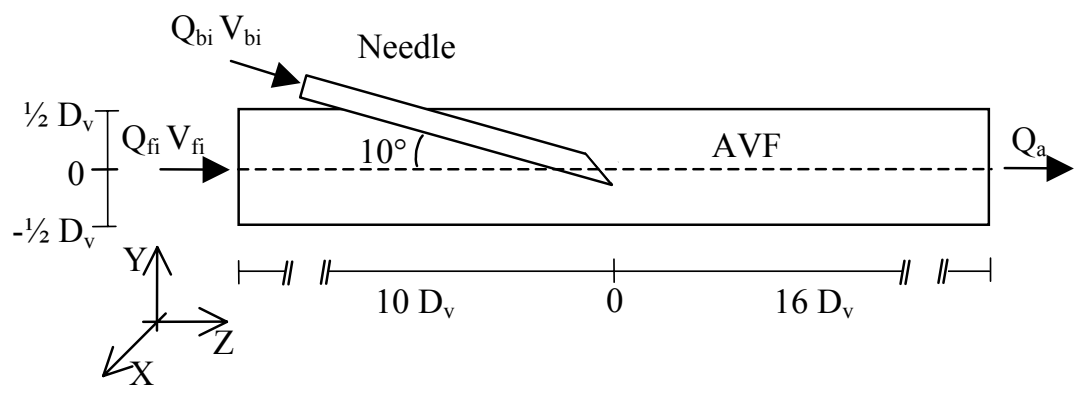

Figure 1: The symmetry plane $(\mathrm{x}=0)$ view of the venous needle inserted in an artero-venous fistula (AVF). Dimensions are not to scale. Symbols used: $\mathrm{D}_{\mathrm{v}}$ diameter of AVF, Qa access flow, $\mathrm{Q}_{\mathrm{bi}}$ and $\mathrm{V}_{\mathrm{bi}}$ needle flow and velocity, $\mathrm{Q}_{\mathrm{fi}}$ and $\mathrm{V}_{\mathrm{fi}}$ inlet $\mathrm{AVF}$ flow and velocity. 
The needle dimensions employed in this study (16 Gauge needle Plume-S, Hospal, Italy), were $1.4 \mathrm{~mm}$ inner diameter and $1.6 \mathrm{~mm}$ external diameter. The needle was assumed to be rigid in this study. The AVF was modelled as a cylindrical tube with rigid walls and a constant diameter $\left(D_{v}\right)$ of $6.5 \mathrm{~mm}$. AVF diameter was derived clinically from an echographic analysis of a dialytic patient (Nephrology Division, San Carlo Clinic, Paderno Dugnano (Mi), Italy). The total axial length of the model was $169 \mathrm{~mm}$ with $65 \mathrm{~mm}$ upstream $\left(10 * \mathrm{D}_{\mathrm{v}}\right)$ and 104 $\mathrm{mm}\left(16^{*} \mathrm{D}_{\mathrm{v}}\right)$ downstream of the needle. The upstream and downstream lengths were chosen to minimise the computational entrance and exit effects. The needle was inserted such that an angle of $10^{\circ}$ was formed between the axis of the needle and the AVF axis. In this study blood was considered to be a homogenous, incompressible and Newtonian fluid with a viscosity and density of $1055 \mathrm{~kg} / \mathrm{m}^{3}$ and $3.6 \mathrm{e}^{-3} \mathrm{~Pa} \mathrm{~s}$ respectively. These assumptions have been shown to be reasonable in previous studies $[5,6]$.

\subsection{Model solution and blood flow boundary conditions}

A 3-D computational model was built to reproduce the venous needle insertion in the AVF (see Fig. 1) using the CFD pre-processor Gambit (v2.0.4 - Fluent Inc., Lebanon, $\mathrm{NH}$ ). A symmetry assumption was used to reduce the computational effort by simulating blood flow in one half of the geometry. An unstructured grid composed of tetrahedral mesh elements was generated using the TGRID meshing scheme. The flow boundary conditions used in this study included velocity inlet condition both at the needle inlet (fully developed profile) and the AVF inlet (flat profile), a traction free outflow condition, a symmetry condition and a rigid wall condition. CFD simulations were carried out with different re-infusion blood flows $\left(\mathrm{Q}_{\mathrm{bi}}\right)$ and post withdrawal arterial flows $\left(\mathrm{Q}_{\mathrm{fi}}\right)$ that matched the patient access flow rate $\left(\mathrm{Q}_{\mathrm{a}}=500 \mathrm{ml} / \mathrm{min}\right)$. $\mathrm{Q}_{\mathrm{a}}$ was measured invivo by Transonic $\AA\left(H D 01^{\text {plus }}\right.$, Transonic Systems Inc., Ithaca, NY, USA) based on the Krivitsky method [7]. The flow values in the AVF inlet $\left(\mathrm{Q}_{\mathrm{fi}}\right)$ were imposed as follows:

$$
Q_{f i}=Q_{a}-Q_{b i} \text { with } Q_{b i}=0,50,100,150,200,250,300 \mathrm{ml} / \mathrm{min}
$$

The arterial withdraw was assumed equal to $\mathrm{Q}_{\mathrm{bi}}$, thus no ultrafiltration in dialyzer was involved. Different velocity conditions $\mathrm{V}_{\mathrm{bi}}$ and $\mathrm{V}_{\mathrm{fi}}$ used in this study are presented in Tab. 1.

\subsection{Numerical method}

The commercial CFD solver Fluent (v6.1.18 - Fluent Inc., Lebanon, NH) was used to simulate the re-infusion of blood in AVF during hemodialysis treatment. Fluent used a finite volume technique to solve the 3-D steady equations of momentum and mass conservation. The mass and momentum transport equations were solved using the QUICK scheme and the pressure-velocity coupling was achieved through the SIMPLE scheme. The mesh independence of solutions was 
investigated by successively refining the grid by increasing the number of nodes by a factor of approximately two. Computational solutions obtained with three different mesh densities (371,504, 155,043 and 86,113 nodes) were then compared against each other. Mesh independence was considered to have been achieved when the maximum difference between the pressure in the AVF axis and on the outlet face of the needle between successive simulations was less than $5 \%$. Based on these criteria, a mesh containing 155,043 nodes was chosen as the optimum case for simulations.

Table 1: Velocities and the corresponding Reynolds number used in this study.

\begin{tabular}{|c|c|c|c|c|c|}
\hline Case & $\begin{array}{c}\text { Flow } \mathrm{Q}_{\mathrm{bi}} \\
{[\mathrm{ml} / \mathrm{min}]}\end{array}$ & $\begin{array}{c}\text { Mean velocity } \\
\mathrm{V}_{\mathrm{bi}}[\mathrm{m} / \mathrm{s}]\end{array}$ & $\begin{array}{c}\text { Mean velocity } \\
\mathrm{V}_{\mathrm{fi}}[\mathrm{m} / \mathrm{s}]\end{array}$ & $\begin{array}{c}\mathrm{Re} \\
\mathrm{Q}_{\mathrm{bi}}\end{array}$ & $\begin{array}{c}\mathrm{Re} \\
\mathrm{Q}_{\mathrm{fi}}\end{array}$ \\
\hline $\mathrm{A}$ & 0 & 0 & 0.25 & 0 & 476 \\
\hline $\mathrm{B}$ & 50 & 0.52 & 0.225 & 213 & 428 \\
\hline $\mathrm{C}$ & 100 & 1.04 & 0.2 & 426 & 380 \\
\hline $\mathrm{D}$ & 150 & 1.56 & 0.175 & 640 & 333 \\
\hline $\mathrm{E}$ & 200 & 2.08 & 0.15 & 853 & 285 \\
\hline $\mathrm{F}$ & 250 & 2.6 & 0.125 & 1066 & 238 \\
\hline $\mathrm{G}$ & 300 & 3.12 & 0.1 & 1280 & 190 \\
\hline
\end{tabular}

\section{Results}

The CFD simulation results of the venous needle insertion in an AVF (see Fig. 1) are presented. The computational results are primarily concerned with the description of velocity and wall shear stress field under different re-infusion $\left(\mathrm{Q}_{\mathrm{bi}}\right)$ and inlet AVF $\left(\mathrm{Q}_{\mathrm{fi}}\right)$ flow conditions. Convergence difficulties were encountered for the $F$ and $G$ cases because of high needle flow rates $\left(Q_{b i}\right)$. This could be related to the assumption of laminar flow model.

\subsection{Velocity results}

The different values of blood flow in the needle $\left(\mathrm{Q}_{\mathrm{bi}}\right)$ imposed during the simulations led to the prediction of different velocity fields in the region downstream to the needle tip. Fig. 2 presents the axial velocity field downstream of the needle in the symmetry plane for the Case G. In all the simulations except when $\mathrm{Q}_{\mathrm{bi}}$ was zero, the reinfusing flow stream was found to hit the bottom wall of the AVF. As expected the intensity of the impact was found to increase with $\mathrm{Q}_{\mathrm{bi}}$. Also shown in Fig. 2 is the appearance of ripples near the bottom wall of Case $\mathrm{G}$ due to the high flow rate $\left(\mathrm{Q}_{\mathrm{bi}}=300 \mathrm{ml} / \mathrm{min}\right)$. This phenomenon occurred only in the cases $\mathrm{F}$ and $\mathrm{G}$ (Fig. 3). In the upper part of the AVF, a local recirculation of blood with negative $z$ axial velocity was noticed. A localized zone of turbulence appeared downstream of the needle tip $\left(1 \mathrm{D}_{\mathrm{v}}<\mathrm{z}<5 \mathrm{D}_{\mathrm{v}}\right)$ between region with z-velocity positive and negative one. The z-velocity patterns were significantly different during the cases simulated. Fig. 3 demonstrates the 
influence of $\mathrm{Q}_{\mathrm{bi}}$ and $\mathrm{Q}_{\mathrm{fi}}$ on $\mathrm{z}$ axial velocity profiles in the symmetry plane downstream of the needle for the cases $\mathrm{C}, \mathrm{E}$, and $\mathrm{G}$. These profiles were evaluated at the same plane shown in Fig. $2\left(-\mathrm{D}_{\mathrm{v}} / 2<\mathrm{y}<\mathrm{D}_{\mathrm{v}} / 2\right.$ and $\mathrm{z}=0, \mathrm{D}_{\mathrm{v}}, 2 \mathrm{D}_{\mathrm{v}}, 4$ $\left.D_{v}\right)$. For all the cases simulated, the maximum value of $z$ axial velocity in the AVF was located at the injection point of $Q_{b i}$ in the AVF (see Fig. 3, panel $z=0$ ). At this location, the $\mathrm{z}$ axial velocity was found to increase by about $50 \%$ more than the $\mathrm{V}_{\mathrm{bi}}$. In the Case $\mathrm{G}$ case, blood velocity of $4.5 \mathrm{~m} / \mathrm{s}$ which is almost fortyfive times higher than $V_{f i}$ was noticed. In general the velocity gradient between $\mathrm{V}_{\mathrm{fi}}$ and $\mathrm{V}_{\mathrm{bi}}$ and the different flow direction of the main axial and the reinfusing blood caused the onset of turbulent flow patterns in the AVF. This led to the appearance of local recirculation regions in the upper part of the AVF. In particular, this happened only when $\mathrm{Q}_{\mathrm{bi}}$ was greater than $200 \mathrm{ml} / \mathrm{min}$ (that is, $40 \%$ of $Q_{a}$, see cases E, F, and G). In fact, as shown in Fig. 3, mainly in case G, downstream to the needle $\left(z=0, D_{v}, 2 D_{v}\right.$ and $\left.4 D_{v}\right)$, the velocity was negative in the upper part of AVF and positive in the lower part.

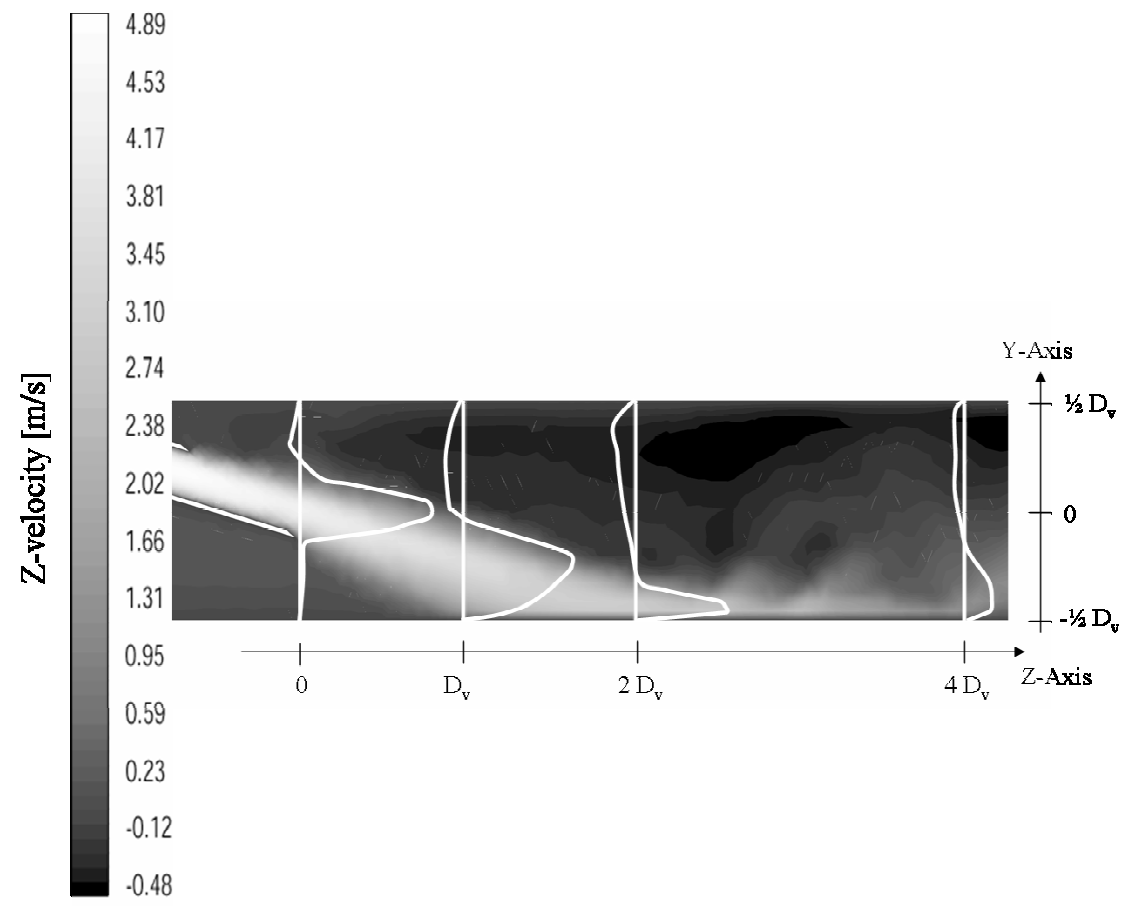

Figure 2: Axial $(Z)$ velocity patterns in the symmetry plane $(x=0)$ for the Case $G$. $D_{v}$ represents the length of the AVF diameter.

The point of reversal of velocity was found to be dependent upon axial $(Z)$ value and on the values of $\mathrm{V}_{\mathrm{fi}}$ and $\mathrm{V}_{\mathrm{bi}}$ (Fig. 3). For instance, for case $\mathrm{E}$, the velocity became negative only on the upper half of the tube at $z=4 D_{v}$. However, in Case $G$, the velocity was negative at all the axial locations shown in 
Fig. $3\left(z=0, D_{v}, 2 D_{v}, 4 D_{v}\right)$ especially in the upper half of the tube. In contrary, for case $C$, the axial velocity profiles were always positive there was no evidence of reverse flow. In the worst case simulated (Case $\mathrm{G}$ ), the maximal value of negative velocity was about $-0.5 \mathrm{~m} / \mathrm{s}\left(\mathrm{z}=4 \mathrm{D}_{\mathrm{v}}\right)$, a value five times higher than $\mathrm{V}_{\mathrm{fi}}$.
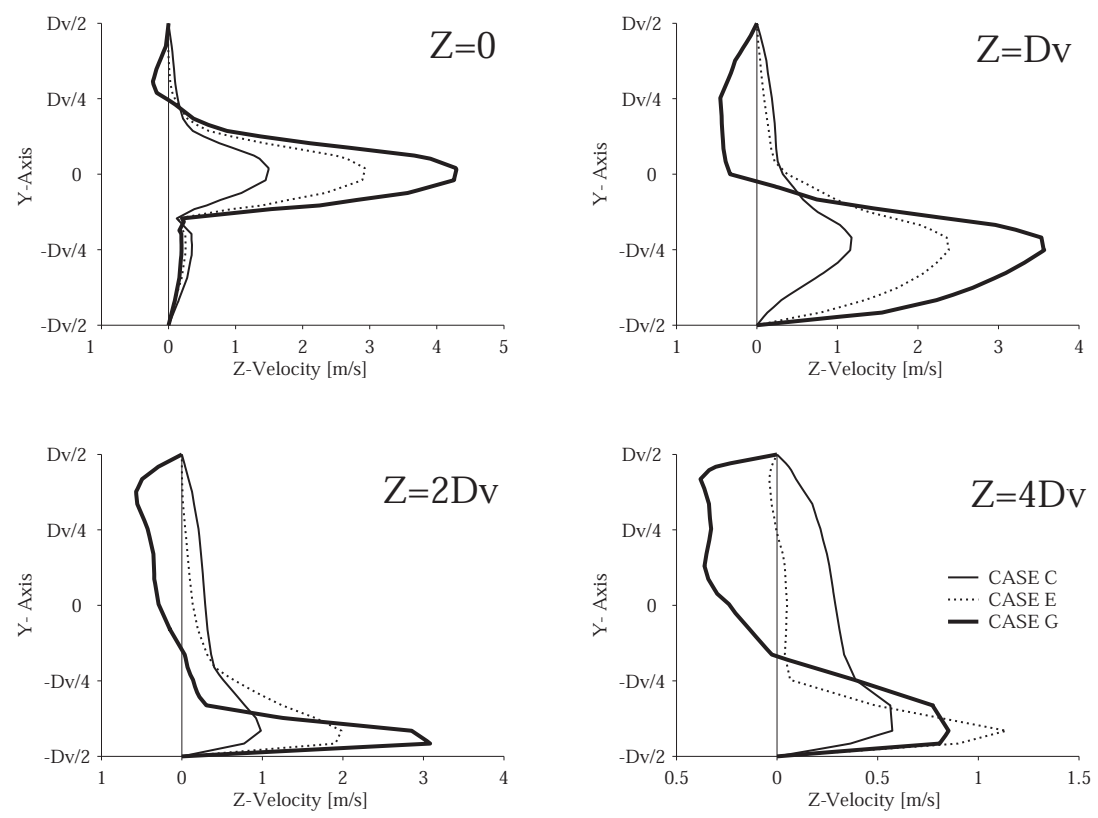

Figure 3: Axial $(Z)$ velocity profiles downstream of the needle $\left(z=0, D_{v}, 2 D_{v}\right.$ and $4 \mathrm{D}_{\mathrm{v}}$ ). $\mathrm{D}_{\mathrm{v}}$ represents the AVF diameter.

\subsection{Shear stress results}

The value of wall shear stress due to the impact of $Q_{b i}$ jet on the bottom part of the AVF wall was evaluated in all the cases simulated (see Table. 1). In particular, zones of the AVF wall with a wall shear stress value higher than 40 $\mathrm{Pa}$ was considered as critical for the formation of aneurysms as reported in previous studies $[2,3]$. The extent of this critical high shear zone was assessed by computing the wall shear stress values along the bottom wall ( $x=0$ and $y=-1 / 2$ $\mathrm{D}_{\mathrm{v}}$, see Fig. 1) in the symmetry plane. As expected, in the cases with high $\mathrm{Q}_{\mathrm{bi}}$ values (Cases D, E, F and G) high shear zones were found to form in the critical zone. In fact, the shear stress on the AVF wall was less than $40 \mathrm{~Pa}$ for the simulations with $\mathrm{Q}_{\mathrm{bi}}$ less than $150 \mathrm{ml} / \mathrm{min}$ (Cases A, B and C). The shear stress values and the critical zone were markedly different during the simulations with $\mathrm{Q}_{\text {bi }}$ ranging from $150 \mathrm{ml} / \mathrm{min}$ to $300 \mathrm{ml} / \mathrm{min}$ (see Fig. 4). The calculated peak values were 44, 64, 90 and $114 \mathrm{~Pa}$ for the cases D, E, F and G, respectively. The 
length of AVF with wall shear values higher than $40 \mathrm{~Pa}$ was significantly different in the cases $\mathrm{D}$ and $\mathrm{E}\left(0.7^{*} \mathrm{D}_{\mathrm{v}}\right.$ vs. $\left.2.1^{*} \mathrm{D}_{\mathrm{v}}\right)$. However, in the cases $\mathrm{F}$ and $\mathrm{G}$, the extent of critical zone was similar, (around $3 * \mathrm{D}_{\mathrm{v}}$ ) even if the average value of shear stress in Case G was 20\% higher than Case F (59 Pa vs. $71 \mathrm{~Pa}$ ).

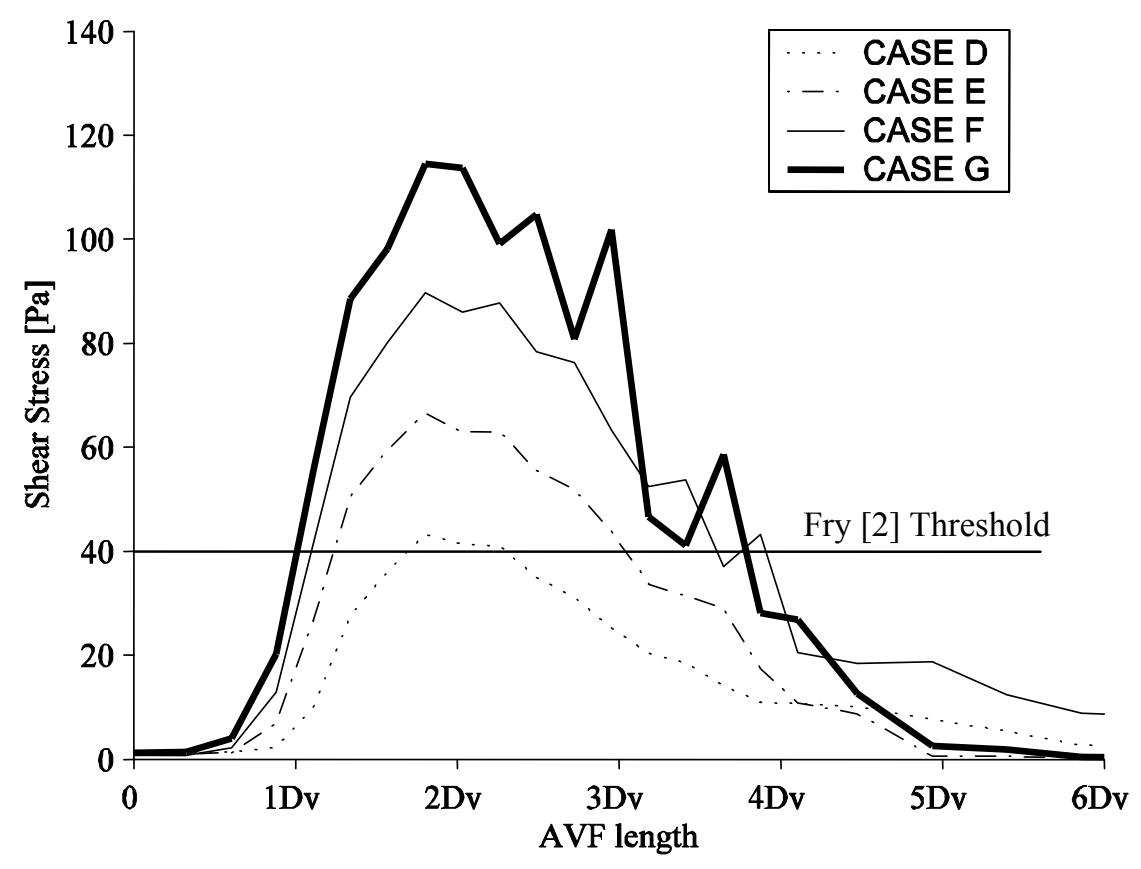

Figure 4: Shear stress evaluated on the bottom symmetry line $(x=0$ and $y=$ $-D_{\mathrm{v}} / 2$, see Fig. 1) of AVF wall during different simulation cases (see Tab. 1).

\section{Discussion}

A local hemodynamic analysis of the point of venous return in AVF was carried out by the application of CFD. This provided a clear understanding of the critical conditions due to the injection of blood along a hemodialysis needle. To reproduce realistic vessel geometry, the AVF diameter measured experimentally by echographic analysis of a dialytic patient was used. The dimensions of the vessel were similar to previous analyses on dialytic patients $[8,9]$. In our case the simulations were done with the needle tip located on the axis of the AVF, inserted at an angle of 10 degrees to the AVF axis. This angle was imposed as a result of geometric analysis of real needle insertion. The needle site within the vessel was also chosen based on a realistic situation in which the risk of contact between the AVF walls and the cutting part of the hemodialysis needle is minimized. 
The blood was modelled as a homogenous, incompressible and Newtonian fluid with constant density and viscosity as considered in previous studies $[5,6]$. A laminar flow model was used to simulate the pressure and velocity field in the AVF. In the simulations with Reynolds number higher than 1000 (Case F and G) it was difficult to achieve convergence. This suggests that the laminar flow model used is perhaps not suited for simulating high Reynolds number flows. This warrants future studies with a suitable turbulent flow model.

The velocity field simulated showed the appearance of turbulent flow patterns with a reverse blood flow regions in the cases where the reinfusion flow rate was higher than $200 \mathrm{ml} / \mathrm{min}$ (cases E, F and G). The reverse flow was limited to a region few centimeters downstream of the needle. In physiological situations, due to blood flow pulsatility the zone of retrograde flow may shift upstream or downstream of the locations predicted in this study and could potentially lead to critical recirculation. The velocity field predicted in this study was similar to that predicted Borg and Funchs in an in vitro model [10]. In particular, the CFD velocity fields were similar to the mixing patters observed in their study.

The CFD model was also used to analyze the shear stress on the AVF walls. The simulations highlighted that the impact of the blood flow on the bottom wall of the fistula involved a not physiological level of shear stress. In fact, high values of shear stress were individuated in the zone of impact between $\mathrm{Q}_{b i}$ and the AVF wall. The values of shear stress on the AVF wall were in accordance with a study by Borg [3], who highlighted not physiological values in arterovenous fistula. According to Fry [2], the structure of endothelial cells can be damaged by shear stress higher than $40 \mathrm{~Pa}$, if this stress is continuous as during the hemodialysis treatment. In line with this hypothesis, the simulation predicted a higher increase of critical zone by changing the reinfusion blood flow. The effect of the injection of blood by the venous needle and the related shear stress is surely linked to the design of the needle and on its position on the AVF. The needle site is a parameter that during hemodialysis treatment can not be precisely monitored. The dialysis machine, indeed, gives information only if the needle tip is too near to AVF wall, usually with a negative pressure alarm. The experience of the clinicians together with an echographic exam during treatment can help individuating the needle site. Therefore the risk of endothelial damage can be decreased from one side with an accurate design of the needle and from the other one by monitoring the blood flow values related to the vascular access and its geometrical characteristics.

\section{References}

[1] Feldman H. I., Kobrin S., Wasserstein A., Hemodialysis vascular access morbidity. J. Am Soc Nephrol. 7, pp. 523-535, 1996.

[2] Fry D. L., Acute vascular endothelial changes associated with increased blood velocity gradients. Circ. Res. 22, pp. 165-197, 1968.

[3] Borg A., Experimental and numerical studies on flow related to the processes of atherosclerosis. ISRN LUTMDN/MVK-1022-SE, Ph.D. Thesis, Lund Institute of Technology, Sweden, 2000. 
[4] Mareels G., De Wachter D. S. \& Verdonck P. R., Computational Fluid Dynamics-Analysis of the Niagara Hemodialysis Catheter in a Right Heart Model. Artif. Organs 28(7), pp. 639-648, 2004.

[5] Dash R. K., Jayaraman G. \& Metha K. N., Flow in catheterized artery with stenosis. J. Biomech 32(1), pp. 49-61, 1999.

[6] Grigioni M., Daniele C., Morbiducci U., D’Avenio G., Di Benedetto G., Del Gaudio C. \& Barbaro V., Computational model of the fluid dynamics of a cannula inserted in a vessel: incidence of the presence of side holes in blood flow. J. Biomech 35, pp. 1599-1612, 2002.

[7] Krivitsky N. M., Theory and validation of access flow measurement by dilution technique during hemodialysis. Kidney Int., 48, pp. 244-250, 1995.

[8] De Wachter D. \& Verdonck P., Numerical Calculation of Hemolysis Levels in Peripheral Hemodialysis Cannulas. Artif. Organs 26(7), pp. 576-582, 2002

[9] Van Tricht I., De Wachter D., Vanhercke D., Tordoir J. \& Verdonck P., Assessment of Stenosis in Vascular Access Grafts. Artif. Organs 28(7), pp. 617-622, 2004.

[10] Borg A. \& Fuchs L., LIF study of mixing in a model of vein punctured by a cannula. Int. J. of Heat Fluid Flow 23, pp. 664-670, 2002. 